\title{
A Research on Novel Human Resource Model
}

\author{
Yanda Li \\ North China Electric Power University, Baoding 071003, China \\ yanda325221@163.com
}

Keywords: Resource Model; stochastic simulation; Novel Human.

\begin{abstract}
In this paper, two simulation models are built to describe the dynamic change of human resources and come to some conclusions on which the great decisions of HR manager are based. First of all, we introduce the personnel structural model on the basis of stochastic simulation. We analyze the churn, promotion and recruitment of staff and obtain different personnel structures by changing the rate of churn. Next, we adopt the personnel attribute model which reflects the changes of individual attribute in a dynamic process. Especially in terms of employee's satisfaction, we use BP neural network as an acquisition method and depict dynamic characteristics of attribute by using personnel relationship network at the same time. Finally, we analyze sensibility of the model. At the same time, we discuss the merits and demerits of the model in detail.
\end{abstract}

\section{Introduction}

We should confirm HR dynamic procedureconsists of two layers.One is the alteration of staff structure, that is to say, the change of different stratums in number. Another is the change of staff attribute, such as the change of staffs satisfaction.Generally speaking, these two kinds of changes are supportive with each other. So as to simplify the question, we set up two models to reect them. For the former, we mainly discuss how to describe the alteration of unsure staff structure authentically. We can use stochastic simulation and roulette [1] selection to analyze it. For the latter, the method to gain the staff attribute should be given firstly, then consider the dynamic changes of the attribute.

According to the team science and the multi-network theory, both models can be combined and the best team performance can be obtained in the case of having complete information.

\section{Assumption}

1. We assume ICM personnel structure refers to the number of employees under each level rather than the intrinsic rigid structure in which each department is regarded as a unit.

2. We assume the changes of personnel structure only comprises churn, promotion and recruit. Other factors are not considered.

\section{Model}

\subsection{The Personnel Structure Model}

We take into account that the dynamic process of ICM personnel resource network refers to the personnel structure change along with the time in the first place.Although the personnel structure change is certain in practical circumstance, it is difficult to abstract realistic circumstance to the certain mathematical model directly. In order to describe the personnel structure change veritably as much as possible, we consider to use the idea of stochastic simulation to analyze the problem.

Churn. The total churn number of the i level in ICM per year $\left(N_{i}\right)$ can be measured by the parameter $P_{i}$. To simplify analysis, we define the following contents:

$$
N_{i}=w_{i} \cdot P_{i}
$$

Thereof, $w_{i}$ is on behalf of the rated personnel number of per level. We use the idea of stochastic simulation to pre-determine the churn number of various levels in per month; 


$$
\sum_{j=1}^{12} n_{i j}=N_{i}
$$

\subsection{Personnel Attribute Model}

Attribute acquisition. In terms of the specific staff in the enterprise, hard targets are easy to acquire and promotion rates from fixed target mapping is relatively clear, for example, we can take expert assessment strategies directly for the promotion chance of each person. By contrast, soft targets are a bit complicated, so we focus on soft targets to consider the ways of obtaining and measuring in the following discussions.

Referring to literature [2], we consider that the employees for business satisfaction degree is mainly relevant to the factors listed in Table 1. Using Table 1, enterprise management can investigate each staff to get raw data. We can adopt BP neural network to analyze because the impact on the satisfaction of each indicator is uncertain. First, the actual situation of the company in the past or similar to the companys data network is trained; then use the trained network, by entering the values of the investigation factors, to get everyones satisfaction index.

Using BP neural network to acquire staff satisfaction degree can be seen in Fig.2 and Fig.3.

Table 1: Business factors score sheet on employee loyalty

\begin{tabular}{|c|c|}
\hline Business factors & Meanings \\
\hline Number & \\
\hline Emolument & \\
\hline Communication Degree & \\
\hline Prospects & \\
\hline Enterprise Culture & \\
\hline Work Environment & \\
\hline Management Level & \\
\hline Employee Loyalty & \\
\hline
\end{tabular}

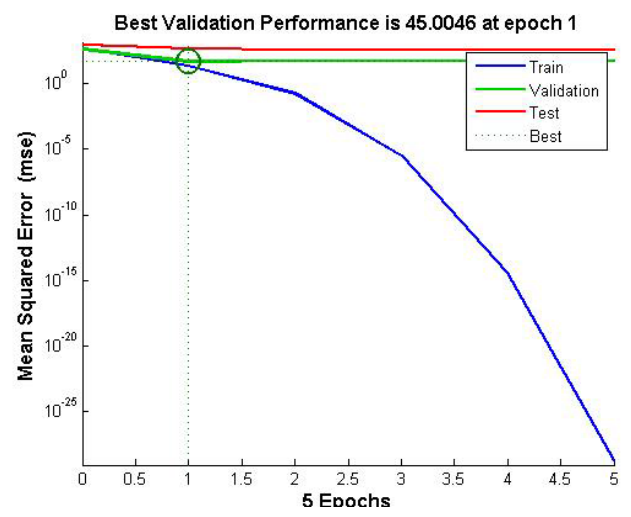

Figure 2: Training Performance
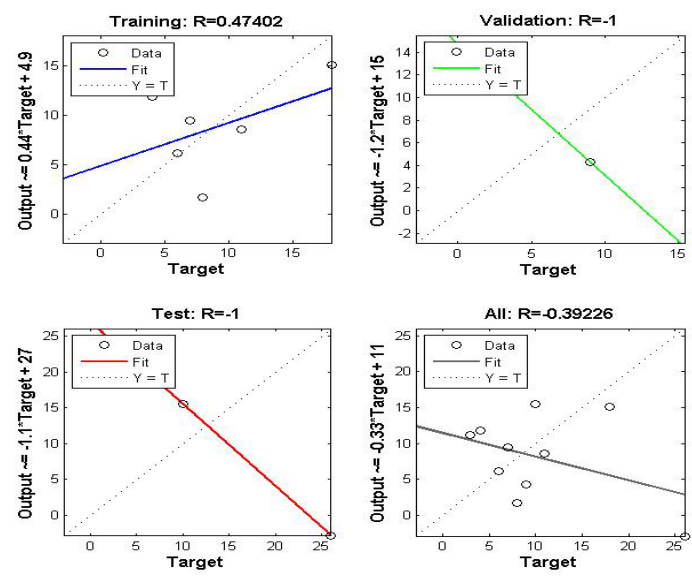

Figure 3: Training Regression 
Conclusion. In general, in cases where the reasonable assumptions are satisfied, basing on the idea of stochastic simulation, we quantitatively described the dynamic changes of personnel structure following various churn rate and, combining the employees cognition model, analyzed comprehensively the influence of personnel cognitive level (such as satisfaction degree) to the internal personnel alteration situation. On such a basis, we got some ideas for HR Manager.

\section{Testing the Model}

Sensibility Analysis. According to the introduction of recruit referred in the question, we know that ICM will fill about 2/3 of the vacancies after integrating administrative delays and office and internal promotions and so on. In this regard, we make a sensitivity calibration by changing the parameter in a small range. Through quantitative analysis, we find that the organizations promotion rate is reasonable compared with the churn rate when we try to refill about $2 / 3$ of the vacancy .At the same time, we find that it has a little effect on model results when the parameters change within the range of $-0: 05$. It proves that our model has a high stability.

\section{Strengths and Weaknesses}

\subsection{Strengths}

Based on the above analysis, our model is reasonable and can simplify the question that we discuss greatly.

\subsection{Weaknesses}

Throughout the analysis process, we do not consider the dynamic personnel change circumstance.

\section{References}

[1] http://wenku.baidu.com/view/b9b5fe6ba98271fe910ef9c6.html.

[2] Qi Zeng, Mingming Liu, Jun Xu. The basis analysis of employee loyalty based on BP netural network. Page 61 to Page 62.

[3] E. Salas, N.J. Cooke, and M.A. Rosen. (2008). On Teams, Teamwork, and Team Performance: Discoveries and Developments. Human Factors: The Journal of the Human Factors and Ergonomics Society June 2008 vol. 50 no. 3 540-547.

[4] D. Stokols, K.L. Hall, B.K. Taylor, R.P. Moser (2008). The Science of Team Science: Overview of the Field and Introduction to the Supplement, Am J Prev Med 2008;35(2S): S77-S89.

[5] Mikko Kivel a, Alexandre Arenas, Marc Barthelemy, James P. Gleeson, Yamir Moreno, Mason A. Porter. (2013). Multilayer Networks, J. Complex Networks, 2(3): 203-271 (2014); arXiv preprint arXiv:1309.7233, 2013. 